\title{
A New GM(1,1) Model Based on Cubic Monotonicity-Preserving Interpolation Spline
}

\author{
Yuanpeng Zhu ${ }^{1, *}$, Zehua Jian ${ }^{2}$, Yurui Du ${ }^{2}$, Wenqing Chen ${ }^{2}$ and Jiwei Fang ${ }^{2}$ \\ 1 School of Mathematics, South China University of Technology, Guangzhou 510640, China \\ 2 School of Business Administration, South China University of Technology, Guangzhou 510640, China; \\ bmjzh@mail.scut.edu.cn (Z.J.); bmgreeny@mail.scut.edu.cn (Y.D.); bmcwq@mail.scut.edu.cn (W.C.); \\ bmjwfang@mail.scut.edu.cn (J.F.) \\ * Correspondence: ypzhu@scut.edu.cn
}

Received: 28 February 2019; Accepted: 19 March 2019; Published: 21 March 2019

\begin{abstract}
In the classical GM(1,1) model, an accumulated generating operation is made on the original non-negative sequence to obtain a monotone increasing 1-AGO sequence, and the forecasting model is established based on the 1-AGO sequence. A great number of scholars have improved the accuracy of grey model prediction through better developed background value and the equation for the time response. In this work, we reconstruct the background value based on a new developed monotonicity-preserving piecewise cubic interpolations spline, and thereby establish a new GM(1,1) model. Numerical examples show that the new $\mathrm{GM}(1,1)$ model has better prediction quality of data than the original GM(1,1) model and improves the precision of prediction in practice.
\end{abstract}

Keywords: GM(1,1); grey theory; background value; monotonicity-preserving

\section{Introduction}

Let an original non-negative and uniformly spaced sequence be

$$
X^{(0)}=\left\{x^{(0)}(1), x^{(0)}(2), \cdots, x^{(0)}(n)\right\} .
$$

The main idea of the classical grey forecasting GM(1,1) model proposed by Deng [1,2] is to make an accumulated generating operation on the original sequence, so as to reduce the randomization of the original data and obtain an obviously monotone increasing 1-AGO sequence $X^{(1)}$. Then, establish a first-order grad forecasting differential equation on the sequence $X^{(1)}$. In addition, further use the least-square method to numerically solve the differential equation to estimate the parameters. Finally, the original data simulation and prediction are carried out by using the inverse accumulated generating operation.

The 1-AGO sequence $X^{(1)}$ is given as follows

$$
X^{(1)}=\left\{x^{(1)}(1), x^{(1)}(2), \cdots, x^{(1)}(n)\right\},
$$

where

$$
x^{(1)}(k)=\sum_{i=0}^{k} x^{(0)}(k)=x^{(1)}(k-1)+x^{(0)}(k), k=1,2, \cdots, n .
$$


From Equation (3), we can see that the 1-AGO sequence $X^{(1)}$ has the property of monotonicity-increasing. Suppose that $x^{(1)}(t)$ meets the following first-order grad forecasting differential equation

$$
\frac{d x^{(1)}(t)}{d t}+a x^{(1)}(t)=b
$$

where the grey developmental coefficient $a$ and the grey control parameter $b$ are the parameters in the model to be estimated.

The solution of the Equation (4) with the initial condition $\widetilde{X}^{(1)}(1)=X^{(1)}(1)$ is as follows

$$
x^{(1)}(t)=\left[x^{(1)}(1)-\frac{b}{a}\right] e^{-a(t-1)}+\frac{b}{a} .
$$

Therefore, to obtain the prediction model of the raw data sequence, we need to identify the effect of the grey development coefficient $a$ and the grey control parameter $b$ in Equation (4). For this purpose, we do the integral accumulation on both sides of Equation (4) for $[k, k+1], k=1,2, \cdots, n-1$, then we can get

$$
\int_{k}^{k+1} \frac{d x^{(1)}(t)}{d t} d t+a \int_{k}^{k+1} x^{(1)}(t) d t=b,
$$

that is

$$
x^{(1)}(k+1)-x^{(1)}(k)+a \int_{k}^{k+1} x^{(1)}(t) d t=b,
$$

or

$$
x^{(0)}(k+1)+a \int_{k}^{k+1} x^{(1)}(t) d t=b .
$$

Let background value be $z^{(1)}(k+1):=\int_{k}^{k+1} x^{(1)}(t) d t$. To calculate the background value $z^{(1)}(k+1)$, we need to integrate $x^{(1)}(t)$, which requires the values of $a$ and $b$ to be given in advance. However, the values of $a$ and $b$ need to be determined from the Equation (6). Consequently, to estimate the values of $a$ and $b$, we must use some methods to estimate the background value $z^{(1)}(k+1)$. We use the piecewise linear polynomial interpolation $L(t):=(k+1-t) x^{(1)}(k)+(t-k) x^{(1)}(k+1)$ to approximate $x^{(1)}(t)$ in the classical GM(1,1) model, see [1,2], then we get the estimated background value $z^{(1)}(k+1)$ as follows

$$
\begin{aligned}
z^{(1)}(k+1) & =\int_{k}^{k+1} x^{(1)}(t) d t \\
& \approx \int_{k}^{k+1} L(t) d t \\
& =\frac{1}{2}\left[x^{(1)}(k)+x^{(1)}(k+1)\right] .
\end{aligned}
$$

For each interval $[k, k+1], k=1,2, \cdots, n-1$, by substituting the estimated background value $z^{(1)}(k+1)$ into Equation (6) and further applying the least-square method, we estimate the values of the parameters $a$ and $b$ by the formula as follows

$$
\left(\begin{array}{l}
a \\
b
\end{array}\right)=\left(G^{T} G\right)^{-1} G^{T} X,
$$


where

$$
X=\left[\begin{array}{c}
x^{0}(2) \\
x^{0}(3) \\
\vdots \\
x^{0}(n)
\end{array}\right], G=\left(\begin{array}{cc}
-z^{(1)}(2) & 1 \\
-z^{(1)}(3) & 1 \\
\vdots & \vdots \\
-z^{(1)}(n) & 1
\end{array}\right)
$$

Finally, we get the following estimated solution to the differential Equation (4) with the initial condition $\widetilde{X}^{(1)}(1)=X^{(1)}(1)$ as follows

$$
\widetilde{x}^{(1)}(t)=\left[x^{(1)}(1)-\frac{b}{a}\right] e^{-a(t-1)}+\frac{b}{a}, k=1,2, \ldots
$$

We thus get the following grey prediction equation

$$
\widetilde{x}^{(0)}(k+1)=\widetilde{x}^{(1)}(k+1)-\widetilde{x}^{(1)}(k), k=1,2, \ldots
$$

From (7), it can be found that the classical GM(1,1) model uses average of adjacent values to estimate the background value $z^{(1)}(k+1)$. Its geometric meaning is to use the area of straight ladder to replace the trapezoidal area which is based on the edge of exponential curve $x^{(1)}(t)$, as shown in Figure 1. This method has a shortcoming that when the 1-AGO data sequence varies greatly, the result of prediction will have large error $(\Delta S)$ with the exponential increasing. Thus, if we apply the classical GM $(1,1)$ model in practice, there exist some restrictions. As pointed out in [3-5], the accuracy of prediction in $\operatorname{GM}(1,1)$ model depends on the estimation of the background value $z^{(1)}(k+1)$. In [6], $\mathrm{Li}$ and Dai reconstructed $x^{(1)}(t)$ by a high-order Newton interpolation polynomial. In addition, they estimated the background value $z^{(1)}(k+1)$ based on the Newton-Cores integral. However, as shown in [6], when there is a mass of data, the high-order Newton interpolation polynomial may have the Runge phenomenon and thus the truncation error may be very large. Furthermore, the numerical stability is not guaranteed when calculating the approximate value of Newton-Cores integral. In [7], Tang and Xiang estimated the background value $z^{(1)}(k+1)$ by using the piecewise quadratic interpolation polynomial to reconstruct $x^{(1)}(t)$ on the interval $[k, k+1]$. The advantage is that it has the characteristics of less computation and good numerical stability. In [8], Wang et al. used piecewise cubic interpolation spline to reconstruct $x^{(1)}(t)$ and thus got the estimated background value $z^{(1)}(k+1)$. The advantages of the given method are that it can avoid the Runge phenomenon resulted from high-order polynomial. However, the methods we mentioned above all ignore the important monotonicity-increasing feature of the curve $x^{(1)}(t)$ to be reconstructed. If the resulting reconstructed curve $x^{(1)}(t)$ loses the monotonicity-increasing feature, there will also result in a large error on the background value $z^{(1)}(k+1)$. Therefore, an accurate approximation of the reconstructed curve $x^{(1)}(t)$ is the key to enhance the estimation of the background value.

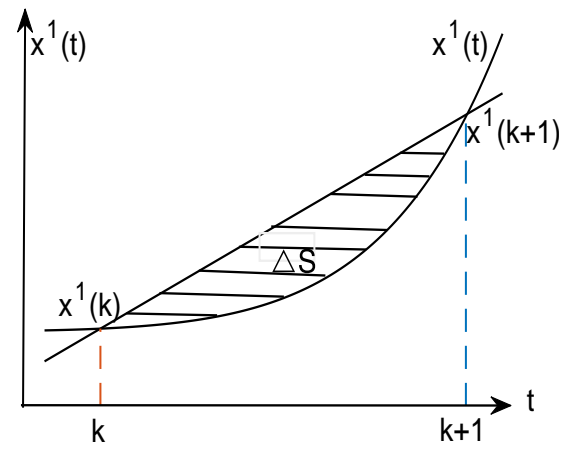

Figure 1. Prediction error source diagram of conventional GM(1,1) model. 
Recently, lots of scholars proposed many methods to improve the classical GM(1,1) model. Liu and his colleagues have proposed some new methods of grey development clustering based on the multiattribute difference, which enriches the grey fixed-weight cluster theory, see [9-11] for example. In [12-14], Xie and his colleagues proposed some new grey models, including Verhulst model and the segmental corrected new information discrete GM(1,1) model. In [15], Yang and his colleagues presented a dynamic $\mathrm{GM}(1,1)$ model based on grey system theory and cubic spline function interpolation principle. Many scholars have put many efforts on the establishments of new grey prediction models, see for example [16-31] and the references therein.

In this paper, we shall propose a monotonicity-preserving piecewise cubic interpolation spline to reconstruct the curve $x^{(1)}(t)$ and thereby give a new scheme to estimate the background value $z^{(1)}(k+1)$. The rest of this paper is structured as follows. Section 2 represents the construction of the $C^{1}$ monotonicity-preserving cubic interpolation splines. In Section 3, based on $C^{1}$ monotonicity-preserving cubic interpolation spline, a new GM(1,1) model is constructed in detail. Several numerical examples are also given. In addition, the conclusion is given in Section 4.

\section{2. $C^{1}$ Monotonicity-Preserving Piecewise Cubic Interpolation Spline}

According to Equation (3), the 1-AGO sequence $X^{(1)}$ has the property of monotonicity-increasing, that is $x^{(1)}(k) \leq x^{(1)}(k+1), \forall k$. The fitting exponential curve $x^{(1)}(t)$ to the 1-AGO sequence $X^{(1)}$ is also monotonicity-increasing and has infinite smoothness. Therefore, we develop a $C^{1}$ monotonic-preserving cubic interpolation spline to interpolate the 1-AGO sequence, to reconstruct the curve $x^{(1)}(t)$.

For the discrete data $\left(k, x^{(1)}(k)\right), k=1,2, \ldots, n$, we denote $d^{(1)}(k)$ as the derivative value at node $t=k$. For $t \in[k, k+1]$, a cubic interpolation spline with local parameter $\alpha_{k}$ is constructed as follows

$$
\begin{aligned}
B(t)= & (1-s)^{3} x^{(1)}(k)+3(1-s)^{2} s\left[x^{(1)}(k)+\frac{d^{(1)}(k)}{\alpha_{k}}\right] \\
& +3(1-s) s^{2}\left[x^{(1)}(k+1)-\frac{d^{(1)}(k+1)}{\alpha_{k+1}}\right]+s^{3} x^{(1)}(k+1),
\end{aligned}
$$

where $s=t-k \in[0,1]$. From Equation (10), after some computations, we have

$$
\left\{\begin{array}{l}
B(k)=x^{(1)}(k), B(k+1)=x^{(1)}(k+1), \\
B^{\prime}(k)=\frac{3 d^{(1)}(k)}{\alpha_{k}}, B^{\prime}(k+1)=\frac{3 d^{(1)}(k+1)}{\alpha_{k+1}},
\end{array}\right.
$$

which indicates that $B\left(k^{-}\right)=B\left(k^{+}\right), B^{\prime}\left(k^{-}\right)=B^{\prime}\left(k^{+}\right)$. This means that the cubic interpolation spline defined by Equation (10) is $C^{1}$ continuous for arbitrary nonzero local parameter. Here, $C^{1}$ continuity means that a function together with its first-order derivative function is continuous. In addition, it is of interest to note that for all $\alpha_{k}=3$, the cubic interpolation spline given in Equation (10) returns into the classic cubic Hermite interpolation spline.

In practical application, we should estimate the derivative values of the cubic interpolation spline at the nodes at first. In this paper, we calculate the derivative values by the following method

$$
\left\{\begin{array}{l}
d^{(1)}(1)=x^{(1)}(2)-x^{(1)}(1), \\
d^{(1)}(k)=\frac{1}{2}\left[x^{(1)}(k+1)-x^{(1)}(k-1)\right], \quad k=2,3, \ldots, n-1, \\
d^{(1)}(n)=x^{(1)}(n)-x^{(1)}(n-1) .
\end{array}\right.
$$


We shall derive sufficient conditions for the $C^{1}$ cubic interpolation spline preserving monotonicity. For the monotonicity-increasing 1-AGO sequence $X^{(1)}$, it is obvious that the derivative value determined by Equation (12) is non-negative, which means $d^{(1)}(k) \geq 0, \forall k$. Without loss of generality, for $t \in[k, k+1]$, direct computation gives that

$$
\begin{aligned}
B^{\prime}(t)= & 3(1-s)^{2} \frac{d^{(1)}(k)}{\alpha_{k}}+s^{2} \frac{d^{(1)}(k+1)}{\alpha_{k+1}} \\
& +6(1-s) s\left\{\left[x^{(1)}(k+1)-x^{(1)}(k)\right]-\left[\frac{d^{(1)}(k)}{\alpha_{k}}+\frac{d^{(1)}(k+1)}{\alpha_{k+1}}\right]\right\} .
\end{aligned}
$$

Thus, we can see that the following conditions are sufficient to guarantee $R^{\prime}(t) \geq 0$

$$
\left\{\begin{array}{l}
\alpha_{k}>0, \quad \alpha_{k+1}>0, \\
{\left[x^{(1)}(k+1)-x^{(1)}(k)\right]-\left[\frac{d^{(1)}(k)}{\alpha_{k}}+\frac{d^{(1)}(k+1)}{\alpha_{k+1}}\right]} \\
=\left[\frac{x^{(1)}(k+1)-x^{(1)}(k)}{2}-\frac{d^{(1)}(k)}{\alpha_{k}}\right]+\left[\frac{x^{(1)}(k+1)-x^{(1)}(k)}{2}-\frac{d^{(1)}(k+1)}{\alpha_{k+1}}\right] \geq 0 .
\end{array}\right.
$$

Summarizing the above discussion, we can obtain the following sufficient conditions for $B(t)$ preserving monotonicity

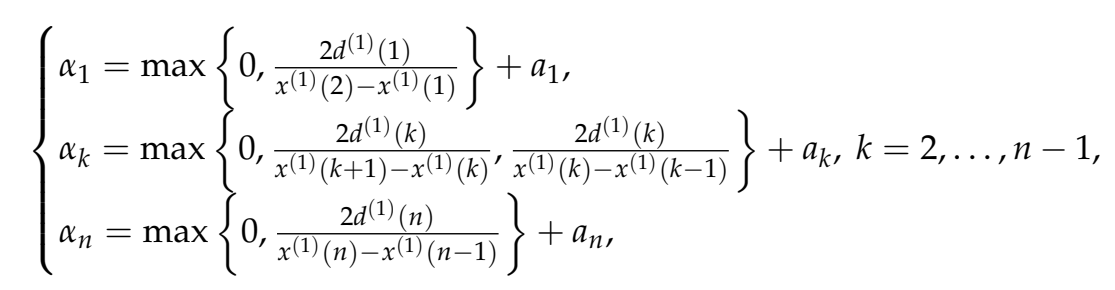

where $a_{i}>0$ serves as free parameter. In practice, we recommend all $a_{k}=3$. If there is no special explanation below, we will take all $a_{k}=3$.

\section{Establish New GM(1,1) Model}

For the original non-negative sequence $X^{(0)}=\left\{x^{(0)}(1), x^{(0)}(2), \cdots, x^{(0)}(n)\right\}$, we first calculate its 1-AGO sequence $X^{(1)}=\left\{x^{(1)}(1), x^{(1)}(2), \cdots, x^{(1)}(n)\right\}$. Then for the 1-AGO sequence $X^{(1)}$, we use the sufficient conditions (14) with all the free parameters $a_{k}=3$ to determine a $C^{1}$ monotonicity-preserving piecewise cubic interpolation spline $B(t)$ to reconstruct the exponential curve $x^{(1)}(t)$. For the interval $[k, k+1]$, we estimate the background value $z^{(1)}(k+1)=\int_{k}^{k+1} x^{(1)}(t) d t$ by the following method

$$
\begin{aligned}
z^{(1)}(k+1)= & \int_{k}^{k+1} x^{(1)}(t) d t \\
\approx & \int_{k}^{k+1} B(t) d t \\
= & \frac{1}{4} x^{(1)}(k)+\frac{1}{4}\left[x^{(1)}(k)+\frac{d^{(1)}(k)}{\alpha_{k}}\right] \\
& +\frac{1}{4}\left[x^{(1)}(k+1)-\frac{d^{(1)}(k+1)}{\alpha_{k+1}}\right]+\frac{1}{4} x^{(1)}(k+1) .
\end{aligned}
$$


Then by substituting the estimated background value into the grey differential Equation (6), we further apply the following least-square method to solve Equation (6)

$$
\left(\begin{array}{l}
a \\
b
\end{array}\right)=\left(G^{T} G\right)^{-1} G^{T} X
$$

where

$$
X=\left[\begin{array}{c}
x^{0}(2) \\
x^{0}(3) \\
\vdots \\
x^{0}(n)
\end{array}\right], G=\left(\begin{array}{cc}
-z^{(1)}(2) & 1 \\
-z^{(1)}(3) & 1 \\
\vdots & \vdots \\
-z^{(1)}(n) & 1
\end{array}\right)
$$

Finally, we get the following estimated solution to the differential Equation (4) with the initial condition $\widetilde{X}^{(1)}(1)=X^{(1)}(1)$ as follows

$$
\widetilde{x}^{(1)}(t)=\left[x^{(1)}(1)-\frac{b}{a}\right] e^{-a(t-1)}+\frac{b}{a} .
$$

We thus get the following grey prediction equation

$$
\begin{aligned}
\widetilde{x}^{(0)}(k+1) & =\widetilde{x}^{(1)}(k+1)-\widetilde{x}^{(1)}(k) \\
& =\left(1-e^{a}\right)\left[x^{(1)}(1)-\frac{b}{a}\right] e^{-a k}, k=1,2, \ldots
\end{aligned}
$$

We shall give several examples to show that the new $\operatorname{GM}(1,1)$ model based on $C^{1}$ monotonicity-preserving piecewise cubic interpolation spline has better predict accuracy than the classical GM(1,1) model. In the following examples, the relative error is computed by

$$
\varepsilon=\frac{\left|\bar{x}^{(0)}(k)-x^{(0)}(k)\right|}{x^{(0)}(k)} .
$$

Example 1. In this example, we use the exponential function $f(t)=\eta e^{\lambda t}$ in [20] to generate the original data with convexity. By letting the parameters $g$ and $k$ take a fixed value respectively, the original data sequence can be obtained when the parameter $t$ takes different values. In addition, we compare the results predicted by our new $G M(1,1)$ model with the GM(1,1) model and the method proposed in [20]. The results show that our new model achieves the best results among the three prediction models and it performs very well in predicting data with the exponential growth trend, see Table 1. On the left of Figure 2, the figure shows the 1-AGO data of Table 1 and the curves of piecewise linear interpolant, monotonic-preserving cubic interpolation spline $B(t)$ and the reconstruct exponential curve $X^{(1)}(t)$. From the right Figure 2, we can see that the interpolation spline $B(t)$ has $C^{1}$ continuity.

Table 1. Numerical results for Example 1.

\begin{tabular}{ccccccc}
\hline \multirow{2}{*}{$x^{(\mathbf{0})}$} & \multicolumn{2}{c}{ Classical GM(1,1) } & \multicolumn{2}{c}{ New GM(1,1) } & \multicolumn{2}{c}{ The Model in [20] } \\
\cline { 2 - 7 } & Prediction Data & $\varepsilon \mathbf{( \% )}$ & Prediction Data & $\varepsilon$ (\%) & Prediction Data & $\varepsilon$ (\%) \\
\hline 2.9836 & 2.9836 & 0 & 2.9836 & 0 & 2.9836 & 0 \\
4.4511 & 4.3804 & 1.5816 & 4.3531 & 2.2021 & 4.4561 & 0.1123 \\
6.6402 & 6.5006 & 2.0903 & 6.5222 & 1.7835 & 6.6132 & 0.4066 \\
9.9061 & 9.6469 & 2.5994 & 9.7720 & 1.3569 & 9.8146 & 0.9237 \\
14.7781 & 14.3162 & 3.1039 & 14.6413 & 0.9344 & 14.5657 & 1.4373 \\
22.0464 & 21.2454 & 3.6069 & 21.9368 & 0.5013 & 21.6168 & 1.9486 \\
32.8893 & 31.5285 & 4.1065 & 32.8675 & 0.0793 & 32.0812 & 2.4570 \\
\hline $\bar{\varepsilon}(\%)$ & 2.8481 & \multicolumn{5}{c}{1.1429} \\
\hline
\end{tabular}




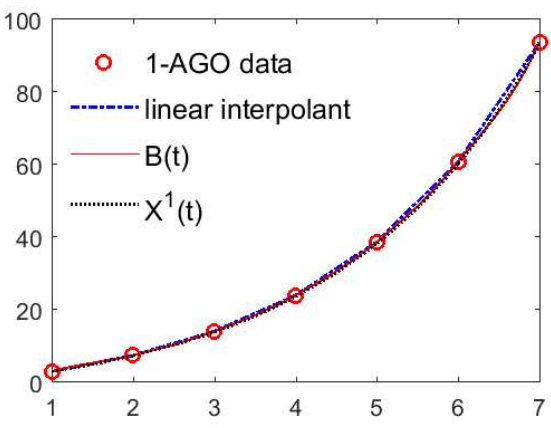

(a) Curves for 1-AGO data.

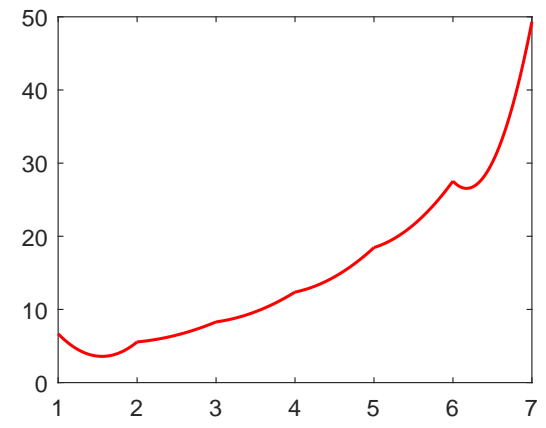

(b) First derivative of $B(t)$.

Figure 2. Graphic results for Example 1.

Example 2. In this example, we directly use the numerical example provided in [21]. We compare our new $G M(1,1)$ model with the classical $G M(1,1)$ model and the prediction models presented in [21]. The results turn out that our new $G M(1,1)$ model prediction accuracy is significantly higher than the classical GM(1,1)model. While the method proposed in [21] performs the best in this example. The reason we suppose is that the method given in [21] simulates and predicts the sequence of exponential distribution by optimizing the background value of the grey differential equations, so that the model has better simulation and prediction accuracy. Table 2 and Figure 3 give the numerical results.

Table 2. Numerical results for Example 2.

\begin{tabular}{ccccccc}
\hline \multirow{2}{*}{$x^{(\mathbf{0})}$} & \multicolumn{2}{c}{ Classical GM(1,1) } & \multicolumn{2}{c}{ New GM(1,1) } & \multicolumn{2}{c}{ The Model in [21] } \\
\cline { 2 - 7 } & Prediction Data & $\varepsilon \mathbf{( \% )}$ & Prediction Data & $\boldsymbol{\varepsilon} \mathbf{( \% )}$ & Prediction Data & $\varepsilon \mathbf{( \% )}$ \\
\hline 21.1 & 21.1 & 0 & 21.1 & 0 & 21.1000 & 0 \\
26.6 & 24.4166 & 8.2083 & 24.0779 & 9.4816 & 23.3606 & 12.1782 \\
36.1 & 35.7198 & 1.0531 & 32.5648 & 9.7928 & 35.7858 & 0.8704 \\
52.3 & 52.2557 & 0.0847 & 50.3116 & 3.8018 & 54.8198 & 4.8180 \\
80.1 & 76.4466 & 4.5611 & 77.7300 & 2.9588 & 83.9777 & 4.8411 \\
126.8 & 111.8361 & 11.8012 & 120.0906 & 5.2913 & 128.6443 & 1.4545 \\
196.3 & 163.6087 & 16.6537 & 185.5365 & 5.4832 & 197.0684 & 0.3914 \\
\hline $\bar{\varepsilon}(\%)$ & 7.0604 & \multicolumn{5}{c}{6.1349} \\
\hline
\end{tabular}

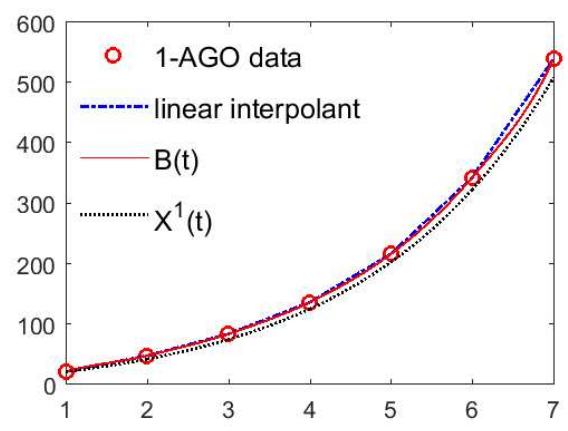

(a) Curves for 1-AGO data.

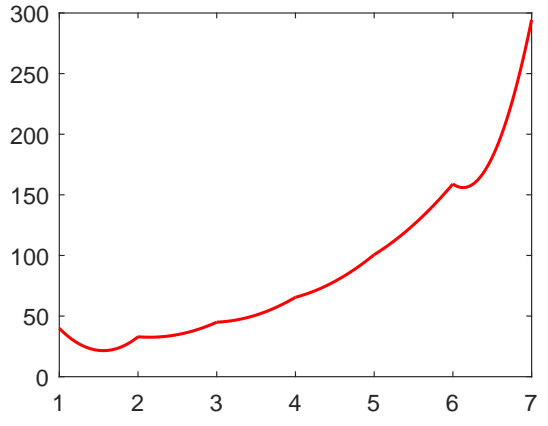

(b) First derivative of $B(t)$.

Figure 3. Graphic results for Example 2.

Example 3. In this example, we use the example in [8] to test the new proposed $G M(1,1)$ model. To verify the applicability of the model to predict electricity consumption in the smart grid, we use the electricity consumption data of Jiangsu province in 2008 into the smart grid as the raw data (in KWh). The results show that the new 
GM $(1,1)$ has improved prediction accuracy compared to the methods proposed in [7] and the classical GM(1,1) model. Table 3 and Figure 4 give the numerical results.

Table 3. Numerical results for Example 3.

\begin{tabular}{ccccccc}
\hline \multirow{2}{*}{$\boldsymbol{x}^{(\mathbf{0})}$} & \multicolumn{2}{c}{ Classical GM(1,1) } & \multicolumn{2}{c}{ New GM(1,1) } & \multicolumn{2}{c}{ The Model in [7] } \\
\cline { 2 - 7 } & Prediction Data & $\boldsymbol{\varepsilon} \mathbf{( \% )}$ & Prediction Data & $\boldsymbol{\varepsilon} \mathbf{( \% )}$ & Prediction Data & $\boldsymbol{\varepsilon} \mathbf{( \% )}$ \\
\hline 110,852 & 110,852 & 0 & 110,852 & 0 & 110,852 & 0 \\
135,175 & 117,980 & 12.72 & 130,078 & 3.77 & 127,821 & 5.41 \\
153,647 & 119,117 & 22.47 & 128,574 & 16.32 & 126,664 & 17.66 \\
120,296 & 128,264 & 6.62 & 127,087 & 5.64 & 125,830 & 4.68 \\
96,362 & 121,422 & 26.27 & 125,617 & 30.36 & 124,380 & 29.23 \\
90,798 & 122,592 & 35.01 & 124,164 & 36.75 & 123,253 & 35.70 \\
102,591 & 123,773 & 20.65 & 122,728 & 19.63 & 122,137 & 19.11 \\
150,534 & 124,965 & 16.99 & 121,308 & 19.41 & 121,031 & 19.63 \\
175,123 & 126,168 & 27.95 & 119,905 & 31.53 & 119,934 & 31.52 \\
127,148 & 113,383 & 10.83 & 118,518 & 6.79 & 114,848 & 9.76 \\
102,085 & 128,610 & 25.98 & 117,147 & 14.75 & 117,772 & 15.47 \\
97,103 & 116,705 & 20.19 & 115,792 & 19.25 & 116,705 & 20.21 \\
\hline $\bar{\varepsilon}(\%)$ & \multicolumn{7}{c}{18.56} \\
\hline
\end{tabular}

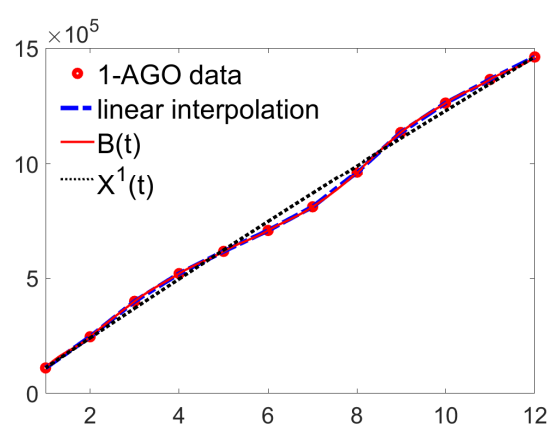

(a) Curves for 1-AGO data.

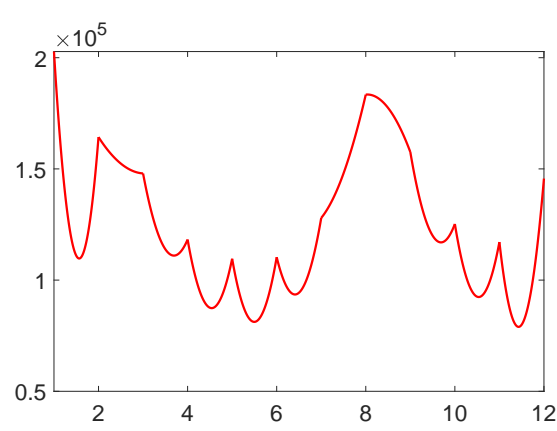

(b) First derivative of $B(t)$.

Figure 4. Graphic results for Example 3.

Example 4. In this example, we select the grey prediction data from [9]. This set of data is based on Chinese health statistics of people with syphilis (in millions) from 2000 to 2010. We compare the new GM(1,1) model with the classical GM(1,1) model and the model in [9]. From Table 4, we can see that our new GM(1,1) model prediction accuracy is improved compared to the classical GM(1,1) model and the model in [9]. Figure 5 gives the graphic results of this example.

Table 4. Numerical results for Example 4.

\begin{tabular}{ccccccc}
\hline \multirow{2}{*}{$x^{(\mathbf{0})}$} & \multicolumn{2}{c}{ Classical GM(1,1) } & \multicolumn{2}{c}{ New GM(1,1) } & \multicolumn{2}{c}{ The Model in [9] } \\
\cline { 2 - 7 } & Prediction Data & $\boldsymbol{\varepsilon} \mathbf{( \% )}$ & Prediction Data & $\boldsymbol{\varepsilon} \mathbf{( \% )}$ & Prediction Data & $\boldsymbol{\varepsilon} \mathbf{( \% )}$ \\
\hline 5.08 & 5.08 & 0 & 5.08 & 0 & 5.08 & 0 \\
4.80 & 3.55 & 26.04 & 4.24 & 11.51 & 3.65 & 23.9 \\
4.67 & 4.52 & 3.21 & 5.25 & 12.57 & 4.61 & 1.28 \\
4.50 & 5.76 & 28.00 & 5.51 & 22.44 & 5.84 & 29.78 \\
7.12 & 7.34 & 3.09 & 8.05 & 13.09 & 7.42 & 4.21 \\
9.67 & 9.34 & 3.41 & 9.97 & 3.05 & 9.45 & 2.28 \\
12.80 & 11.90 & 7.03 & 12.33 & 3.64 & 12.04 & 5.94 \\
15.88 & 15.15 & 4.59 & 15.26 & 3.88 & 15.37 & 3.21 \\
19.49 & 19.30 & 0.97 & 18.89 & 3.07 & 19.65 & 0.82 \\
23.07 & 24.57 & 6.50 & 23.38 & 1.34 & 24.57 & 8.92 \\
26.86 & 31.29 & 16.49 & 28.93 & 7.72 & 31.29 & 19.73 \\
\hline $\bar{\varepsilon}(\%)$ & \multicolumn{7}{c}{7.48} \\
\hline
\end{tabular}




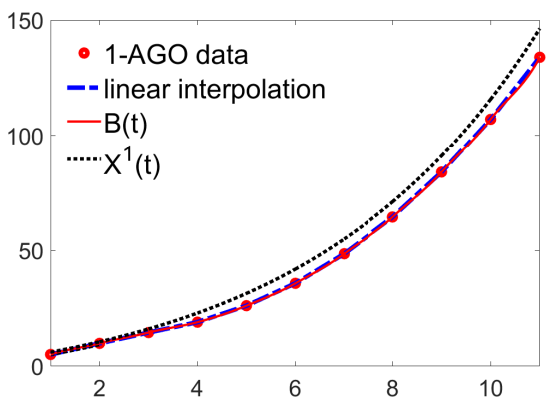

(a) Curves for 1-AGO data.

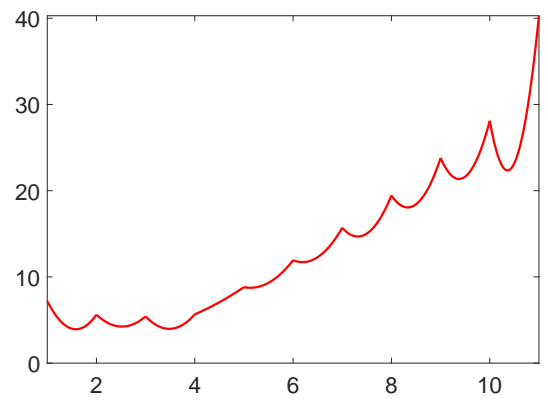

(b) First derivative of $B(t)$.

Figure 5. Graphic results for Example 4.

Example 5. In [11], the data selected by the authors to show a downward trend is very representative, so we use this data sequence in this example. The results show that our new GM(1,1) model is still applicable to the descending sequence, and the accuracy is still slightly improved compared to the classical GM(1,1) model. While the method proposed in [11] performs the best in this example. The reason we suppose is that the method given in [11] simulates and predicts the sequence of exponential distribution by optimizing the background value of the grey differential equations, so that the model has better simulation and prediction accuracy. Table 5 and Figure 6 give the numerical results.

Table 5. Numerical results for Example 5.

\begin{tabular}{ccccccc}
\hline \multirow{2}{*}{$x^{(\mathbf{0})}$} & \multicolumn{2}{c}{ Classical GM(1,1) } & \multicolumn{2}{c}{ New GM(1,1) } & \multicolumn{2}{c}{ The Model in [11] } \\
\cline { 2 - 7 } & Prediction Data & $\varepsilon \mathbf{( \% )}$ & Prediction Data & $\boldsymbol{\varepsilon} \mathbf{( \% )}$ & Prediction Data & $\varepsilon \mathbf{( \% )}$ \\
\hline 1610.71 & 1610.71 & 0 & 1610.71 & 0 & 1610.71 & 0 \\
1245.28 & 1363.91 & 9.524 & 1365.22 & 9.632 & 1342.76 & 7.833 \\
1347.71 & 1274.95 & 5.402 & 1275.75 & 5.340 & 1280.32 & 5.004 \\
1382.45 & 1191.79 & 13.795 & 1192.14 & 13.766 & 1204.58 & 12.872 \\
1018.45 & 1114.06 & 9.381 & 1114.01 & 9.383 & 1122.64 & 10.203 \\
1014.96 & 1041.39 & 2.597 & 1041.00 & 2.566 & 1040.18 & 2.476 \\
949.46 & 973.47 & 2.519 & 972.78 & 2.456 & 960.34 & 1.147 \\
\hline $\bar{\varepsilon}(\%)$ & 6.174 & 6.163 & 5.648 \\
\hline
\end{tabular}

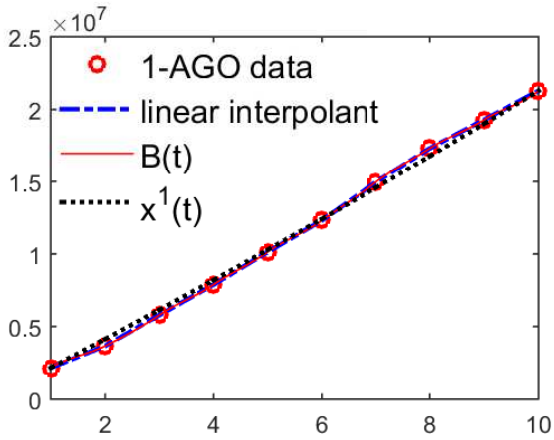

(a) Curves for 1-AGO data.

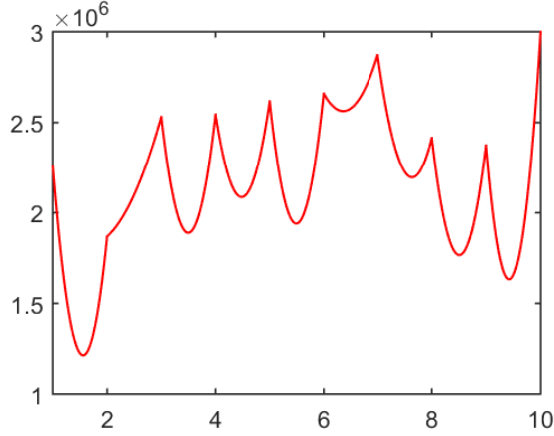

(b) First derivative of $B(t)$.

Figure 6. Graphic results for Example 5.

Example 6. In this example, we selected the relevant data from the 2012 China Statistical Yearbook in [24] to analysis of whether our GM(1,1) model can effectively predict general statistics. Thus, we only compare the prediction results of the classical GM(1,1) model with our new $G M(1,1)$ model. The results show that 
our new GM(1,1) model applies to general sociological statistics, and our model performs better accuracy of prediction compared to classical GM(1,1) model. This shows that our new GM(1,1) model has the ability for further promotion and development. Table 6 and Figure 7 give the numerical results.

Table 6. Numerical results for Example 6.

\begin{tabular}{ccccc}
\hline \multirow{2}{*}{$\boldsymbol{x}^{(\mathbf{0})}$} & \multicolumn{2}{c}{ Classical GM(1,1)} & \multicolumn{2}{c}{ New GM(1,1) } \\
\cline { 2 - 5 } & Prediction Data & $\boldsymbol{\varepsilon} \mathbf{( \% )}$ & Prediction Data & $\boldsymbol{\varepsilon} \mathbf{( \% )}$ \\
\hline $64,832.05$ & $64,832.05$ & 0 & $64,832.05$ & 0 \\
$71,847.09$ & $57,476.77$ & 20.001 & $57,308.50$ & 20.235 \\
$78,646.30$ & $67,165.21$ & 14.598 & $67,022.83$ & 14.779 \\
$86,293.10$ & $78,486.76$ & 9.046 & $78,383.83$ & 9.166 \\
$93,887.95$ & $91,716.70$ & 2.312 & $91,670.62$ & 2.362 \\
$105,557.09$ & $107,176.71$ & 1.534 & $107,209.65$ & 1.566 \\
$125,761.85$ & $125,242.71$ & 0.413 & $125,382.70$ & 0.301 \\
$143,143.63$ & $146,353.96$ & 2.243 & $146,636.24$ & 2.440 \\
$168,850.20$ & $171,023.78$ & 1.287 & $171,492.46$ & 1.565 \\
$198,739.27$ & $199,852.01$ & 0.560 & $200,562.04$ & 0.917 \\
$245,352.80$ & $233,539.60$ & 4.815 & $234,559.18$ & 4.400 \\
$278,541.09$ & $272,905.57$ & 2.023 & $274,319.16$ & 1.516 \\
$334,839.41$ & $318,907.39$ & 4.758 & $320,818.83$ & 4.187 \\
$386,086.72$ & $372,663.28$ & 3.477 & $375,200.63$ & 2.820 \\
\hline $\bar{\varepsilon}(\%)$ & & 4.791 & & 4.732 \\
\hline
\end{tabular}

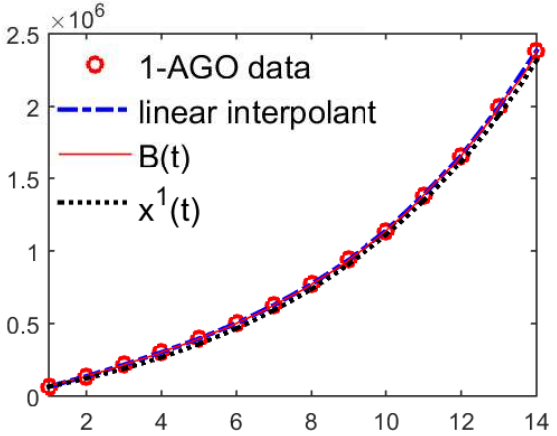

(a) Curves for 1-AGO data.

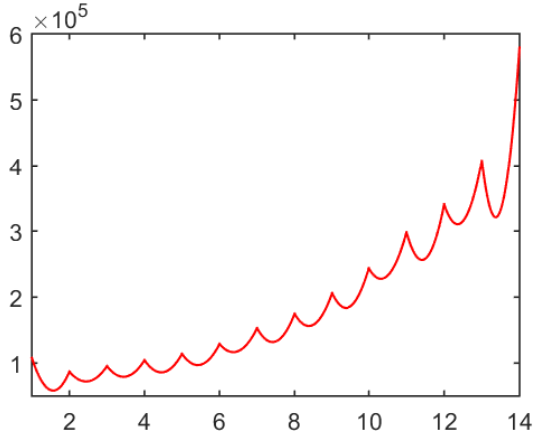

(b) First derivative of $B(t)$.

Figure 7. Graphic results for Example 6.

According to the results of the above numerical examples 1-6, the prediction accuracy of our new $\mathrm{GM}(1,1)$ model is improved for all the numerical examples compared to classical GM(1,1) model. In addition, our new model performs better than the methods proposed in $[7,9,20]$. There are different degrees of improvement for different data features. Based on the above data characteristics, we make the conclusion that the data applicable to our new $\operatorname{GM}(1,1)$ model are generally with the continuously increasing feature over time. In particular, the exponential growth data can show better prediction.

\section{Conclusions}

By using a new developed $C^{1}$ monotonicity-preserving piecewise cubic interpolation spline to reconstruct the background value, we have established a new $\operatorname{GM}(1,1)$ model. Numerical examples show that the new $\mathrm{GM}(1,1)$ model can improve the forecasting quality, especially in prediction reliability and this model performs better when the original data are presented with convexity in time series. Future work will concentrate on exploring more applications of the new $\mathrm{GM}(1,1)$ model, such as scientific decision-making in electricity production and manufactures. 
Author Contributions: Conceptualization, Y.Z. and Z.J.; Methodology, Y.Z. and Z.J.; Software, Y.Z. and Z.J.; Validation, Y.Z.; Formal Analysis, Y.Z.; Investigation, W.C.; Resources, Y.Z. and W.C.; Data Curation, Y.D. and J.F.; Writing-Original Draft Preparation, Y.Z. and Z.J.; Writing-Review \& Editing, Y.Z., Z.J. and Y.D.; Visualization, W.C. and J.F.; Supervision, Y.Z., Z.J. and Y.D.

Funding: This research was funded by the National Natural Science Foundation of China (No. 61802129), the Postdoctoral Science Foundation of China (No. 2015M571931), the Fundamental Research Funds for the Central Universities (No. 2017MS121), the Natural Science Foundation Guangdong Province, China (No. 2018A030310381) and the National Training Program of Innovation and Entrepreneurship for Undergraduates 2018.

Acknowledgments: We thank to the anonymous reviewers for their insightful suggestions and recommendations, which led to the improvements of presentation and content of the paper.

Conflicts of Interest: The authors declare that they have no conflict of interest.

\section{References}

1. Deng, J. Control problems of Grey system. Syst. Control Lett. 1982, 5, 288-294.

2. Deng, J. Grey system basic method. In The Basis of Grey Theory; Julong, D., Ed.; Press of Huazhong University of Science and Technology: Wuhan, China, 2002; pp. 210-313.

3. Tan, G. The structure method and application of background value in grey system GM(1,1) model (I). Syst. Eng. Theory Pract. 2010, 4, 98-103.

4. Tan, G. The structure method and application of background value in grey system GM(1,1) model (II). Syst. Eng. Theory Pract. 2010, 5, 125-132.

5. Tan, G. The structure method and application of background value in grey system GM $(1,1)$ model (III). Syst. Eng. Theory Pract. 2010, 6, 70-74.

6. Li, J.; Dai, W. A new approach of background value building and its application based on data interpolation and Newton-Cotes formula. Syst. Eng. Theory Pract. 2014, 4, 22-126.

7. Tang, W.; Xiang, C. The improvements of forecasting method in $\mathrm{GM}(1,1)$ model based on quadratic interpolation. Chin. J. Manag. Sci. 2006, 14, 109-112.

8. Wang, X.J.; Yang, S.L.; Ding, J.; Wang, H.J. Dynamic GM(1,1) model based on cubic spline for electricity consumption prediction in smart grid. Chin. Commun. 2010, 7, 83-88.

9. Wu, L.; Liu, S.; Yao, L.; Yan, S. The effect of sample size on the grey system model. Appl. Math. Model. 2013, 37, 6577-6583. [CrossRef]

10. Lin, Y.; Ting, Z.; Bingting, Q.; Liu, S. Object multi-attribute differences based grey dynamic clustering method and its application. Oper. Res. Manag. Sci. 2018, 27, 57-63.

11. Wu, L.; Liu, S.; Fang, Z.; Xu, H. Properties of the $\operatorname{GM}(1,1)$ with fractional order accumulation. Appl. Math. Comput. 2015, 252, 287-293. [CrossRef]

12. Cui, J.; Liu, S.; Zeng, B.; Xie, N. Parameters characteristics of grey Verhulst prediction model under multiple transformation. Control Decis. 2013, 28, 605-608.

13. Yao, T.X.; Liu, S.F.; Xie, N.M. Study on the properties of new information discrete GM(1,1) model. J. Syst. Eng. 2010, 25, 164-170.

14. Wang, B.; Xie, N. Unified representation and properties of generalized grey relational analysis models. Syst. Eng. Theory Pract. 2019, 39, 226-235.

15. Hou, L.; Yang, S.; Wang, X. Mid-term load forecasting based on buffer operator and modified grey model. J. Syst. Simul. 2013, 25, 1-5.

16. Fan, J.; Gijbels, I. Local Polynomial Modeling and Its Applications; Chapman and Hall: London, UK, 1996.

17. Bohlin, T. Practical Grey-Box Process Identification: Theory Applications; Springer: Berlin, Germany, 2006.

18. Wang, Z.; Dang, Y.; Liu, S. An optimal GM(1,1) based on the discrete function with exponential law. Syst. Eng. Theory Pract. 2008, 2, 61-67. [CrossRef]

19. Tien, T. A new grey prediction model FGM(1,1). Math. Comput. Model. 2009, 49, 1416-1426. [CrossRef]

20. Wang, Y.; Dang, Y.; Li, Y.; Liu, S. An approach to increase prediction precision of GM(1,1) model based on optimization of the initial condition. Expert Syst. Appl. 2010, 37, 5640-5644. [CrossRef]

21. Wang, Y.; Liu, Q.; Tang, J.; Cao, W.; Li, X. Optimization approach of background value and initial item for improving prediction precision of GM(1,1) model. J. Syst. Eng. Electron. 2014, 25, 77-82. [CrossRef]

22. Liu, J.; Xiao, X.; Guo, J.; Mao, S. Error and its upper bound estimation between the solutions of GM $(1,1)$ grey forecasting models. Appl. Math. Comput. 2014, 246, 648-660. [CrossRef] 
23. Chen, P.; Yu, H. Foundation settlement prediction based on a novel NGM model. Math. Probl. Eng. 2014, 2, 1-8. [CrossRef]

24. He, Z.; Shen, Y.; Li, J.; Wang, Y. Regularized multivariable grey model for stable grey coefficients estimation. Expert Syst. Appl. 2015, 42, 1806-1815.

25. Sidorov, D. Integral Dynamical Models: Singularities, Signals and Control; Vol. 87 of World Scientific Series on Nonlinear Science Series A; World Scientific Publ. Pte Ltd.: Singapore, 2015. [CrossRef]

26. Duan, H.; Xiao, X.; Pei, L. Forecasting the short-term traffic flow in the intelligent transportation system based on an inertia nonhomogenous discrete gray model. Complexity 2017, 2017, 1-16. [CrossRef]

27. Ma, X.; Liu, Z. Application of a novel time-delayed polynomial grey model to predict the natural gas consumption in China. J. Comput. Appl. Math. 2017, 324, 17-24.

28. Wer, B.; Xie, N.; Hu, A. Optimal solution for novel grey polynomial prediction model. Appl. Math. Model. 2018, 62, 717-727. [CrossRef]

29. Wang, Z.; Li, Q.; Pei, L. A seasonal GM(1,1) model for forecasting the electricity consumption of the primary economic sectors. Energy 2018, 154, 522-534. [CrossRef]

30. Zeng, B.; Duan, H.; Bai, Y.; Meng, W. Forecasting the output of shale gas in China using an unbiased grey model and weakening buffer operator. Energy 2018, 151, 238-249. [CrossRef]

31. Wang, J.; Du, P.; Lu, H.; Yang, W.; Niu, T. An improved grey model optimized by multi-objective ant lion optimization algorithm for annual electricity consumption forecasting. Appl. Soft Comput. 2018, 72, 321-337.

(C) 2019 by the authors. Licensee MDPI, Basel, Switzerland. This article is an open access article distributed under the terms and conditions of the Creative Commons Attribution (CC BY) license (http://creativecommons.org/licenses/by/4.0/). 